\title{
Sensibility Study in a Flexible Job Shop Scheduling Problem
}

\author{
Ana Curralo*, Ana I. Pereira*,†, José Barbosa ${ }^{*, * *}$ and Paulo Leitão, ${ }^{*, *}$ \\ *Polytechnic Institute of Bragança, Portugal \\ ${ }^{\dagger}$ ALGORITMI, University of Minho, Portugal \\ ${ }^{*}$ Univ. Lille Nord de France, France \& UVHC, TEMPO research center, France \\ ${ }^{\ddagger}$ LIACC, Portugal
}

\begin{abstract}
This paper proposes the impact assessment of the jobs order in the optimal time of operations in a Flexible Job Shop Scheduling Problem. In this work a real assembly cell was studied: the AIP-PRIMECA cell at the Université de Valenciennes et du Hainaut-Cambrésis, in France, which is considered as a Flexible Job Shop problem. The problem consists in finding the machines operations schedule, taking into account the precedence constraints. The main objective is to minimize the batch makespan, i.e. the finish time of the last operation completed in the schedule.

Shortly, the present study consists in evaluating if the jobs order affects the optimal time of the operations schedule. The genetic algorithm was used to solve the optimization problem. As a conclusion, it's assessed that the jobs order influence the optimal time.
\end{abstract}

Keywords: Flexible Job Shop Problem. Global Optimization. Genetic Algorithm.

PACS: $02.60 . \mathrm{Pn}$

\section{INTRODUCTION}

Life is being rapidly populated by a high amount of offer of products on a daily basis. This offer increase from the industry is also being accompanied side by side, in the consumer perspective, from an increase of product customization and higher quality demands. To cope with these constraints, manufacturing companies must be equipped with more sophisticated production systems [2].

Nowadays, manufacturing systems are seeking for flexibility on their productions, since it provides a higher and faster adaptability, as also robustness increase against perturbations. The flexible manufacturing systems (FMS) are those that are capable to adapt successfully over certain kind of changes in the process environments as well as changing products and process requirements, being able to continue their functions successfully with a certain modified performance [3].

Flexible Job Shop Scheduling Problem (FJSSP) is an extension of the traditional Job Shop Scheduling Problem, differing from this in the sense that some machines may be capable of performing more than one type of job operation. Additionally, for each job operation there is at least, one machine that is capable of performing the operation. The FJSSP may be described as follows: $n$ jobs must be done in a minimum possible time. Each job, $j$, has a unique sequence of operations. The execution of each operation $i$ of job $j, O_{i j}$, requires one machine selected from the machines set. Each machine can process at most one operation at time. The main objective of this work is to find the schedule of the machines, taking into account the precedence constraints, which minimizes the makespan, i.e., the finish time of the last operation completed in the schedule $[1,6,7]$.

Research activities in manufacturing and production control are continually growing and consequently the variety of scheduling and control solutions, having each of them with specific assumptions and possible advantages [6]. However, there are several reasons that prevent that a huge number of scheduling solutions that achieve the stage of industrial implementation or even tests in real conditions. The main difficulty is to provide a robust and reliable performance evaluation of the proposed control systems that would convince industry to take the risk to implement it [6].

This paper addresses a real assembly cell: the AIP-PRIMECA cell at the Université de Valenciennes et du HainautCambrésis, in France, which can be seen as a Flexible Job Shop, leading to the formulation of a FJSSP [3, 2, 6].

Usually, mathematical models are used to study the behavior of manufacturing systems and the difficulty to solve those models results in the high compositional nature, where the amount of variables of the model increases significantly with the growth of the number of products to be produced.

The paper is organized as follows. The first section presents the problem description. The used methodology and the numerical results are presented in the second section and third section, respectively. Finally the last section describes 
the conclusions and future work.

\section{PROBLEM DESCRIPTION}

This work presents a FJSSP formulated from the AIP-PRIMECA Flexible Manufacturing System (FMS), which offers the possibility to create tree different products: "AIP", "LATE" and "BELT". To achieve the products, seven types of jobs that can be manufactured, being denoted by: "B", "E", "L", "T", "A", "I" and "P". For that, it is necessary to assemble, in an appropriate way, a set of components, namely, Plate, Axis_comp, I_comp, L_comp, r_comp and Screw_comp, as shown in Fig. 1.

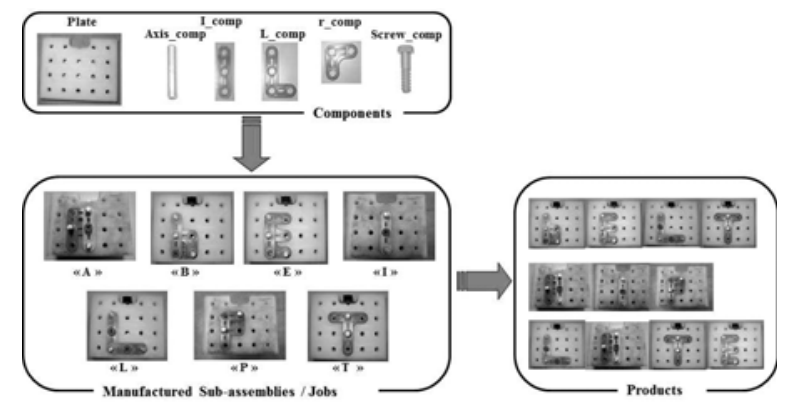

FIGURE 1. Components, jobs and products of the FMS.

The FMS is composed by five workstations, each one being able to perform a set of operations, e.g. assemble the components. A conveyor system serves as transportation between stations, where shuttles are able to transport one product at the time, being released after the product processing conclusion.

In this specific problem, each job has a set of operations that can be done in a set of machines, as described in Table 1. Additionally, it is also assumed that each job, $j$, has an operation list, $O_{. j}$, that needs to be processed in a specific order. Besides this, the operation list also defines the processing times as well the set-up times between operations that are included in processing times. There are eight manufacturing operation types: Plate loading, Axis mounting, r_comp mounting, I_comp mounting, L_comp mounting, Screw_comp mounting, Inspection and Plate unloading. For example, I_comp mounting means that the I component must be mounted on the plate. The inspection is completed by an automatic inspection unit.

TABLE 1. Production sequence for each type of job.

\begin{tabular}{llllllll}
\hline & B & E & L & T & A & I & P \\
\hline$\# 1$ & Plate loading & Plate loading & Plate loading & Plate loading & Plate loading & Plate loading & Plate loading \\
$\# 2$ & Axis mounting & Axis mounting & Axis mounting & Axis mounting & Axis mounting & Axis mounting & Axis mounting \\
$\# 3$ & Axis mounting & Axis mounting & Axis mounting & Axis mounting & Axis mounting & Axis mounting & Axis mounting \\
$\# 4$ & Axis mounting & Axis mounting & Axis mounting & L_comp mounting & Axis mounting & I_comp mounting & r_comp mounting \\
$\# 5$ & r_comp mounting & r_comp mounting & I_comp mounting & r_comp mounting & r_comp mounting & Srewc_comp mounting & L_comp mounting \\
$\# 6$ & r_comp mounting & r_comp mounting & I_comp mounting & Inspection & L_comp mounting & Inspection & Inspection \\
$\# 7$ & I_comp mounting & L_comp mounting & Screw_comp mounting & Plate unloading & I_comp mounting & Plate unloading & Plate unloading \\
$\# 8$ & Screw_comp mounting & Inspection & Screw_comp mounting & & Screw_comp mounting & & \\
$\# 9$ & Inspection & Plate unloading & Inspection & & Inspection & & \\
$\# 10$ & Plate unloading & & Plate unloading & & Plate unloading & & \\
\hline
\end{tabular}

Machines are responsible for the completion of manufacturing operations to do the jobs. Some machines are able to complete the same manufacturing operation, while some manufacturing operations can only be completed on a single machine. Additionally, each machine is continuously available as the system start and each machine can process only one operation at a time. The cell is composed with five machines, specifically:

- $M_{1}$ : loading/unloading unit;

- $M_{2}, M_{3}$ and $M_{4}$ : three assembly workstations;

- $M_{5}$ : automatic inspection unit. 
The processing times of the eight manufacturing operations are described on Table 2.

TABLE 2. Processing time of each manufacturing operation (in seconds).

\begin{tabular}{lccccc}
\hline \multicolumn{1}{c}{ Operation } & $M_{1}$ & $M_{2}$ & $M_{3}$ & $M_{4}$ & $M_{5}$ \\
\hline Plate loading & 10 & & & & \\
Plate unloading & 10 & & & & \\
Axis mounting & & 20 & 20 & & \\
r_comp mounting & & 20 & 20 & & \\
I_comp mounting & & & & 20 & \\
L_comp mounting & & 20 & & 20 & \\
Screw_comp mounting & & & 20 & 20 & \\
Inspection & & & & & 10 \\
\hline
\end{tabular}

\section{PROBLEM FORMULATION AND USED METHODOLOGY}

The problem consists in finding the operations schedule on the machines, taking into account the precedence constraints minimizing the batch makespan, i.e., the finish time of the last operation completed in the schedule.

So, consider $O_{i j}$ the operation $i$ of the job $j$ and $O_{. j}$ represents the set of all operations of the job $j$. Define $l=\max \left\{\left|O_{. j}\right|, j=1, \ldots, n\right\}$ as the maximum number of the operations in a given job and $n$ is the jobs total number.

It is necessary also to define the matrix $M$, with size $l \times n$, representing the machines schedule. $M_{i j}$ represents the machine where the operation $i$ of the job $j$ will be done. The matrix $t$, with size $l \times n$, represents the time schedule. $t_{i j}$ represents the final time of the operation $i$ of the job $j$.

So, it is necessary to solve the following optimization problem:

$$
C_{\text {max }}=\min \left(t_{k n}\right)
$$

where $k$ is the last operation of the last job $n$.

In this work it was intended to solve three "AIP" products, where each product has three jobs. The main question was if the jobs order affect the optimal time. For that it is needed to solve this problem several times, where the difference is the jobs order.

To solve the flexible job shop problem the genetic algorithm (GA) was used. As opposed to many other optimization methods, genetic algorithm works with a population of solutions instead of one single solution. In the GA the solutions are combined to obtain new solutions until a satisfactory solution is obtained. The genetic algorithm is a stochastic method, whose mechanism is based on the simplifications of evolutionary process observed in nature: selection, mutation and crossover.

The GA uses crossover process, where the genes of the best individuals are crossed with genes from other individuals which also have good performance. The algorithm also applies the concept of mutation, thus improving the optimization process by introducing values that were not present in the previous generations. Finally, the genetic algorithm select the best individuals to participate in the next population [4].

\section{NUMERICAL RESULTS}

The numerical results were obtained using a Intel(R) Core(TM) i7 CPU Q720 1.60GHz with 4.0 GB of RAM.

The stop criterion of the GA limits the number of iterations to 100. The number of population individuals was fixed as 30 individuals. Since the GA is a stochastic method, 30 runs were carried out with random initial points.

In the present case, 3 AIP, 9 jobs are created and the jobs order analyzed, assessing its effect in the optimal time solution. For that, 12 combinations of the jobs were tested and the obtained numerical results are presented in Table 3. In this table is indicated the best time of production, $C_{\max }^{\min }$, the average production time of all runs $C_{\max }^{a v g}$ and the average CPU time time avg. 
TABLE 3. Results obtained of the processing time of each product (in seconds).

\begin{tabular}{cccc}
\hline & $C_{\max }^{\min }(s)$ & $C_{\max }^{a v g}(s)$ & $\operatorname{time}^{a v g}(s)$ \\
\hline AAAIIIPPP & 380 & 391 & 26.73 \\
AAAPPPIII & 390 & 409 & 25.99 \\
PPPAAAIII & 400 & 432 & 27.07 \\
PPPIIIAAA & $\mathbf{4 1 0}$ & $\mathbf{4 2 9}$ & 27.22 \\
IIIPPPAAA & 400 & 433 & 25.92 \\
IIIAAAPPP & $\mathbf{3 7 0}$ & $\mathbf{3 8 8}$ & 29.31 \\
AIPAIPAIP & 380 & 403 & 26.43 \\
APIAPIAPI & 380 & 409 & 25.21 \\
PIAPIAPIA & 390 & 424 & 27.02 \\
PAIPAIPAI & 390 & 423 & 26.88 \\
IAPIAPIAP & 380 & 408 & 26.82 \\
IPAIPAIPA & 390 & 421 & 25.70 \\
\hline
\end{tabular}

After the test rounds, is possible to conclude that the jobs order affects the optimal time solution. In the tested problems, the best order was 'IIIAAAPPP' and the worst order was 'PPPIIIAAA'. In the best solution we have the optimal time equal to 370 seconds needing 29.31 seconds to solve the problem.

\section{CONCLUSIONS AND FUTURE WORK}

In this work, the sensitivity of jobs order in a flexible job shop problem problem is analyzed, specifically a batch of 3 AIP products. Twelve problems were solved, where the difference between them were the jobs order. After the tests, it is verified that the jobs order has influence in the optimal result. The best jobs order was 'IIIAAAPPP' with a decrease of $10 \%$ of the $C_{\max }$ considering the worst solution. The difference between the best and the worst solution was 40 seconds in the obtained optimal value.

As future work it is needed to optimize the jobs order of the products and analyze if the jobs order also influence the optimal solution in other type of products. Other optimization methods can be also tested to obtain the optimal solution.

\section{REFERENCES}

1. N. Al-Hinai, T. ElMekkawy, Solving the Flexible Job Shop Scheduling Problem with Uniform Processing Time Uncertainty, World Academy of Science, Engineering and Technology, Vol. 64, pp. 996-1001 (2012).

2. A. Curralo, A. I. Pereira, J. Barbosa and Paulo Leitão, Flexible Job Shop Scheduling Problem in Manufacturing, IO 2013 - XVI Congresso da Associação Portuguesa de Investigação Operacional, pp.70-71 (2013).

3. V. Fernández, Modelling and optimization of flexible manufacturing systems, Instituto Politécnico de Bragança, 2012.

4. M. Kumar, M. Husian, N. Upreti, D. Gupta, Genetic Algorithm: Review and Application, International Journal of Information Technology and Knowledge Management, 2(2), pp. 451-454 (2010).

5. J. Nocedal and S. Wright, Numerical Optimization, Springer Series in Operations Research, Springer, 1999.

6. C. Pach, A. Bekrar, T. Bonte, Y. Sallez, T. Berger, D. Trentesaux, P. Leitão and J. Barbosa, Benchmarking flexible job-shop scheduling and control systems, Control Engineering Practice, Vol. 21, pp. 1204-1225 (2013).

7. R. Zhang, S. Song and C. Wu, A hybrid artificial bee colony algorithm for the job shop scheduling problem, Elsevier - Int. J. Production Economics, Vol. 141, pp. 167-178 (2013). 